\title{
RESEARCH ON ASSEMBLY SEQUENCE PLANNING AND OPTIMIZATION OF PRECAST CONCRETE BUILDINGS
}

\author{
Yaowu WANG ${ }^{1,2}$, Zhenmin YUAN ${ }^{3 *}$, Chengshuang SUN ${ }^{4}$ \\ ${ }^{1}$ Key Lab of Structures Dynamic Behavior and Control of the Ministry of Education, \\ Harbin Institute of Technology, Harbin, 150090, China \\ ${ }^{2}$ Key Lab of Smart Prevention and Mitigation of Civil Engineering Disasters of the Ministry of Industry and \\ Information Technology, Harbin Institute of Technology, Harbin, 150090, China \\ ${ }^{3}$ School of Management, Harbin Institute of Technology, Harbin, 150001, China \\ ${ }^{4}$ School of Economics and Management Engineering, Beijing University of Civil Engineering and Architecture, \\ Beijing, 102616, China
}

Received 27 July 2017; accepted 26 February 2018

\begin{abstract}
Due to more complex structure and increasing prefabrication rate of precast concrete buildings, the assembly order between their constituent components is getting more and more attention. In order to solve the assembly sequence planning and optimization (ASPO) problem in precast concrete buildings, Building Information Modelling (BIM) and Improved Genetic Algorithm (IGA) are organically combined to propose a new method called BIM-IGA-based ASPO method. This method uses BIM for parametric modelling, uses IGA to search for an optimal assembly sequence, and then uses BIM again for visual simulation to further test the assembly sequence. Besides, IGA, which is improved in coding mode, crossover operation and mutation operation, is also used to achieve the dynamic adjustment of assembly sequence in construction process. A full-text example is used to explain the detailed operating principle of BIM-IGA-based ASPO method. The results indicate that the method can effectively find an optimal assembly sequence to reduce the assembly difficulty of a precast concrete building.
\end{abstract}

Keywords: precast concrete buildings, ASPO, BIM, IGA.

\section{Introduction}

After the Second World War, prefabricated buildings were developed rapidly because of their mass production and mechanized construction (Zabihi et al. 2013). Prefabricated buildings in China ever had a development period, but then their development was almost terminated for some reasons (Jiang 2010). The Chinese government actively advocates prefabricated buildings again during the 13th five-year plan, and has made a series of related policies (Liu et al. 2017). Considering the cost, availability and properties of materials, there are three kinds of common prefabricated buildings: wood structure, steel structure and concrete structure (Boafo et al. 2016; Li 2012). As a result of special national conditions, the concrete structure is developed better than the other two structures in China. With the continuous improvement of construction technology, the proportion of precast components in a precast concrete building is increasing (Ding et al. 2016). This makes building houses more like piling up toy bricks or manufacturing cars, and brings a new problem called assembly sequence planning to the construction industry. The problem is becoming more and more prominent in the construction industry. If it cannot be solved well, it may be detrimental to the construction quality, safety, schedule or cost of precast concrete buildings.

The construction mode of precast concrete buildings made them more like manufacturing industry than construction industry. Many efforts have been made to study the assembly sequence planning and optimization (ASPO) of assembly bodies in the manufacturing industry, especially mechanical assembly bodies. Some intelligent algorithms are commonly used to solve the ASPO problem. Qu et al. (2013) determined all feasible assembly sequences of a ship block through constraint reasoning, and combined case-based reasoning with genetic algorithm to find the optimal one from them. Tseng et al. (2010) presented an integrated assembly and disassembly sequence planning

${ }^{*}$ Corresponding author. E-mail: 15b910012@hit.edu.cn 
model based on genetic algorithm. Iwankowicz (2016) established an assembly sequence planning method based on directed acyclic graph and genetic algorithm. Shan et al. (2009) used genetic simulated annealing (GSA) algorithm and ant colony optimization (ACO) algorithm to solve the ASPO problem. Gao et al. (2010) made a comparison among geometric reasoning approach, knowledge-based approach and heuristic method, and found the optimal assembly sequence by a memetic algorithm. Wang and Liu (2010) proposed a chaotic particle swarm optimization approach to plan and optimize the assembly sequence of complex products. In addition, some other methods are also used to solve the ASPO problem. Li et al. (2015) studied the assembly sequence of a ship block from the perspective of disassembly, and utilized analytic hierarchy process (AHP) to determine the best assembly sequence. For reducing computational complexity in assembly sequence planning, Dong et al. (2007) proposed connectionsemantics-based assembly tree (CSBAT) and knowledgebased assembly sequence planning system (KBASP). There are also many similar studies, and they cannot be listed one by one here. Some mature principles in the manufacture industry can be applied to the construction industry (Tezel, Nielsen 2013). Therefore, these methods and theories for mechanical assembly bodies provide reference for the ASPO problem of prefabricated buildings, especially precast concrete buildings.

With the development of prefabricated buildings, the ASPO problem has attracted the attention of some scholars at home and abroad, and they have done some research on it directly or indirectly so far. Some of these studies are aimed at steel structure buildings. Zhao et al. (2015) studied the unloading sequences of a temporary bracing system in large-span steel structures by means of a realcoded genetic algorithm. Gao et al. (2015) put forward a new construction process for rigid bracing dome, which is node-by-node assembling and integral lifting. Some of these studies are aimed at concrete structure buildings. Manrique et al. (2007) gave two factors that affected the assembly sequence of precast concrete buildings, namely space constraints and bracing needs, and used 3D CAD and animation to make a reasonable assembly sequence. Johnston et al. (2016) simulated on-site construction assembly processes by means of virtual prototype (VR). Hu (2007) established the geometric topology diagram between building components, and deduced the disassembly sequence of a precast concrete building so as to obtain its assembly sequence. Shewchuk and Guo (2012) explored the panel-sequencing problem in residential panelised construction without considering the interference between panels, and proposed a solved approach and algorithm. However, relevant research is less according to China Knowledge Resource Integrated Database (CNKI), Engineering Village (EV), Web of Science and Google Scholar. The existing above research on precast concrete buildings has the following problems: (1) Geometric constraints should not be considered too much because the geometric constraints of precast concrete buildings are usually weaker than those of mechanical assembly bodies; (2) BIM has not been well applied although its parametric modelling and visual simulation are better suited for precast concrete buildings; (3) Genetic algorithm is not well used although it has proven to be a very effective method of solving ASPO problem in the manufacturing industry. In order to solve these problems, this paper tries to establish a new method which combines BIM with genetic algorithm and does not pay too much attention to geometric constraints.

This paper associates BIM with Improved Genetic Algorithm (IGA) to study the assembly sequence planning and optimization (ASPO) of precast concrete buildings. This can fully utilize BIM's parameterization and visualization, as well as IGA's advantages in solving optimization problems. First, a new method called BIM-IGA-based ASPO method is created, and a framework flow chart shows its overall operating principle. Especially for IGA, its operation flow is described in detail. Second, take a precast concrete building model as an example, and then establish an index evaluation system and some corresponding mathematical models. On this basis, IGA is used to solve the mathematical models to generate an optimal assembly sequence, and BIM's visual simulation is used to further test the assembly sequence. Besides, this paper also studies the dynamic adjustment of an assembly sequence in construction process, and uses the BIM-IGA-based ASPO method to solve it. Finally, the research is discussed and summarized.

\section{Methodology}

\subsection{Framework flow chart}

BIM-IGA-based ASPO method is an organic combination of Building Information Modelling (BIM) and Improved Genetic Algorithm (IGA). These two techniques in BIMIGA-based ASPO method cooperate with each other so as to better solve the ASPO problem of precast concrete buildings. Figure 1 shows the framework flow chart of BIM-IGA-based ASPO method. BIM, a parametric modelling technique, is used to draw a precast concrete building model which is three-dimensional and visual. Process engineers conduct component classifying and coding on the basis of the model. After the above preparation work is completed, ASPO is started. The expression of an assembly sequence is the premise and key of solving ASPO problem. An assembly sequence is expressed as a continuous and non-repeated positive integer string, and each positive integer of the string represents each component in a precast concrete building. On this basis, establish an evaluation index system, and quantify each index by mathematical methods. So, every feasible assembly sequence has a corresponding value. Then, search for the optimal one from all feasible assembly sequences by IGA which is an improved intelligent algorithm. IGA follows the general process of classic genetic algorithm, but some improvements have been made in some specific steps. The general process of IGA in Figure 1 is as follows: select a coding mode, ini- 


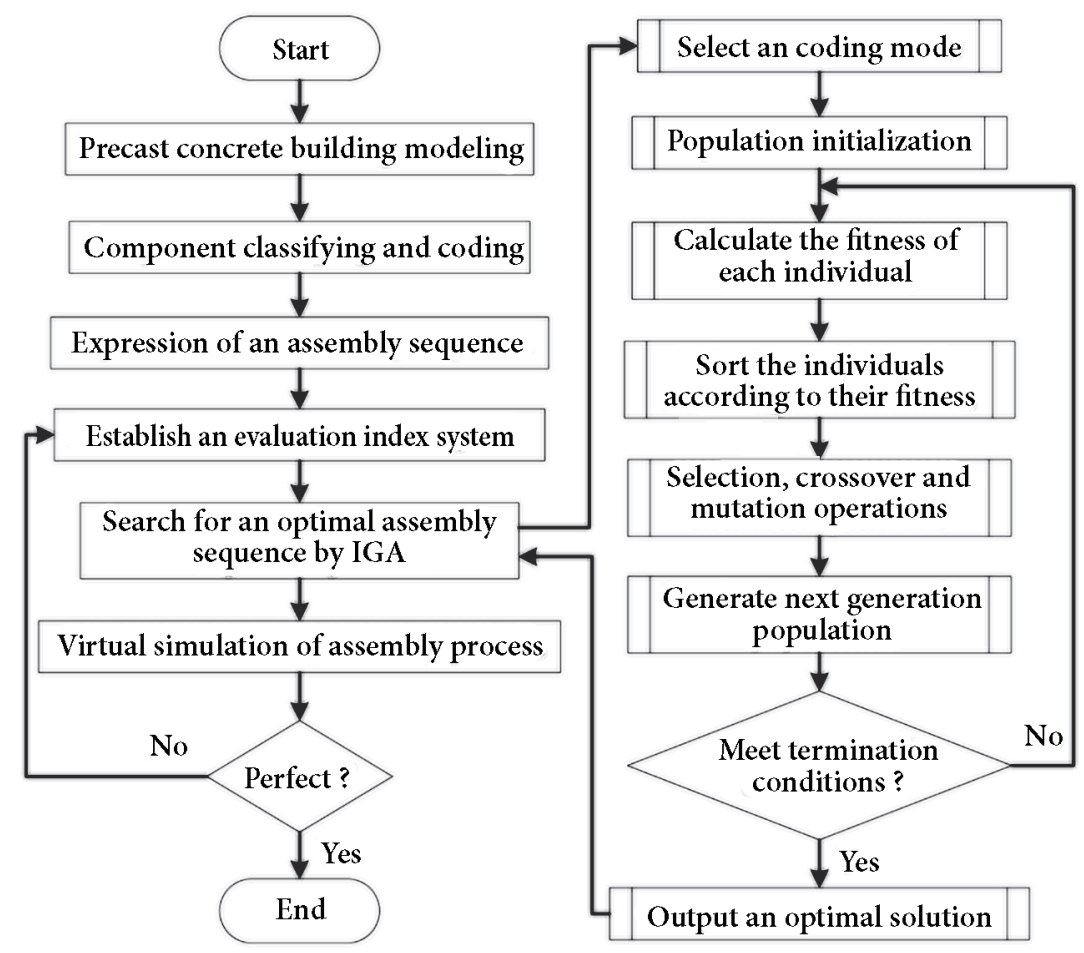

Figure 1. Framework flow chart of BIM-IGA-based ASPO method

tialize population, calculate the fitness of each individual, generate next generation population (selection, crossover and mutation operations), set termination conditions, etc. (Fung et al. 2013; de Oliveira et al. 2014; Han et al. 2014). In order to verify whether the optimal assembly sequence is practical or not, BIM is also again used to simulate the assembly process as real as possible. If it is perfect, then ends; instead, return and continue to search for another optimal assembly sequence.

\subsection{Improved genetic algorithm}

As a kind of common intelligent algorithm, genetic algorithm is based on the natural law of "survival of the fittest" in biological word, and can help decision makers to identify the optimal or near-optimal one from numerous solutions (Aldwaik, Adeli 2014; Senouci, Al-Derham 2008). Its calculation process is generally composed of five steps: select an appropriate coding mode, population initialization, calculate the fitness of each individual, generate next generation population and set termination conditions (Alcaraz, Maroto 2001; Groba et al. 2015). In order to solve the ASPO problem of precast concrete buildings, some improvements should be made in these steps of genetic algorithm. This method is named improved genetic algorithm (IGA). The specific steps of IGA are as follows:

Step 1. Select an appropriate coding mode. A chromosome represents an assembly sequence of a precast concrete building, and the genes in a chromosome represent the components of a precast concrete building. As every component in a precast concrete building owns a unique identification number, real-number encoding is used instead of binary encoding. For a precast concrete building

\begin{tabular}{lc|c|c|c|c|c|c|c|c|}
\hline Genic value & $n-1$ & 1 & 3 & $n$ & 6 & 4 & 2 & 5 & $\cdots$ \\
Genic location & 1 & 2 & 3 & 4 & 5 & 6 & 7 & 8 & $\cdots$
\end{tabular}

Figure 2. Detailed structure of a chromosome

with $n$ components, the genic values in a chromosome take positive integers from 1 to $n$. The detailed structure of a chromosome is shown in Figure 2, including genic location and genic value. It is a continuous and non-repeated positive integer string between 1 and $n$.

Step 2. Population initialization. Population initialization is to generate several chromosomes which meet the rigid constraints between components. The rigid constraints ensure that all generated chromosomes (or assembly sequences) are feasible. However, when the rigid constraints do not exist, every chromosome can be expressed by a string of continuous and non-repeated positive integers between 1 and $n$, and they are all feasible.

Step 3. Calculate the fitness of each individual, and sort the individuals according to their fitness. An individual can be represented by either a chromosome or an assembly sequence. The fitness function is established according to the objective function of assembly sequences. The individuals can be sorted from large to small, or from small to large according to their fitness values.

Step 4. Generate next generation population. The next generation population is generated through the selection, crossover and mutation operations.

(1) Selection operation. Selection operation is to choose better individuals from the previous generation and passes them to the next generation. The size of a population is set according to the actual situation of a problem, which waits 


\begin{tabular}{|c|c|c|c|c|c|c|c|c|c|c|}
\hline Parent chromosome $\mathbf{P}$ & 7 & 1 & 3 & 8 & 6 & 4 & 2 & 5 & $\cdots$ & $n$ \\
\hline \multirow[t]{2}{*}{ Parent chromosome Q } & 1 & 7 & 8 & 4 & 2 & 5 & 6 & 3 & $\cdots$ & $n$ \\
\hline & \multicolumn{10}{|c|}{-5} \\
\hline Parent chromosome $\mathbf{P}$ & 7 & 1 & 3 & 8 & 6 & 4 & 2 & 5 & $\cdots$ & $n$ \\
\hline \multirow[t]{2}{*}{ Parent chromosome Q } & $1^{\prime}$ & 7 & 8 & 4 & 2 & 5 & 6 & -3 & $\cdots$ & $n$ \\
\hline & \multicolumn{10}{|c|}{4} \\
\hline Progeny chromosome $\mathrm{p}$ & 1 & 7 & 8 & 3 & 6 & 4 & 2 & 5 & $\cdots$ & $n$ \\
\hline Progeny chromosome q & 7 & 1 & 3 & 4 & 2 & 5 & 6 & 8 & $\ldots$ & $n$ \\
\hline
\end{tabular}

Figure 3. Detailed principle of improved SPMCM

\begin{tabular}{|c|c|c|c|c|c|c|c|c|c|c|}
\hline Parent chromosome $\mathrm{P}^{\prime}$ & 1 & 7 & 8 & 3 & 6 & 4 & 2 & 5 & $\cdots$ & $n$ \\
\hline$<5$ & \multicolumn{10}{|c|}{$\Delta$} \\
\hline Parent chromosome $\mathrm{P}^{\prime}$ & 1 & 7 & 8 & 3 & 6 & 4 & 2 & 5 & $\cdots$ & $n$ \\
\hline Progeny chromosome $\mathrm{p}^{\prime}$ & 1 & 7 & 4 & 3 & 6 & 8 & 2 & 5 & $\ldots$ & $n$ \\
\hline
\end{tabular}

Figure 4. Detail principle of mutation operation

to be solved. If population size is assumed to be pop, the next generation population generated by selection operation also contains pop individuals. Roulette wheel selection is commonly used to select outstanding individuals from a population (Wang, Chang 2004). Individuals with higher fitness are more opportunities to be selected than those with lower fitness.

(2) Crossover operation. Crossover operation is that the segments at the same position of two chromosomes are interchanged. For the ASPO problem in precast concrete buildings, crossover operation needs to meet the following conditions: (a) inherit excellent segments of parent chromosomes to the greatest extent, (b) all genic values in a progeny chromosome cannot be repeated between each other, (c) the progeny chromosomes meet the rigid constraints. A single point matching crossover method (SPMCM) is established to achieve the above conditions. The detailed principle of SPMCM is shown in Figure 3, and largely draws on partially-mapped crossover (PMX) (Larranaga et al. 1999). First of all, randomly generate one crossover point in the parent chromosomes $\mathrm{P}$ and $\mathrm{Q}$, and define the areas between starting point and crossover point as the matching areas, such as 7-1-3 of parent chromosome $\mathrm{P}$ and 1-7-8 of parent chromosome Q. Then, search for the genes in parent chromosome $\mathrm{P}$ which are the same as 1-7-8 of parent chromosome $\mathrm{Q}$, and record the locations of these genes. Similarly, search for the genes in parent chromosome $\mathrm{Q}$ which are the same as 7-1-3 of parent chromosome $\mathrm{P}$, and record the locations of these genes. Finally, exchange the genes searched in the non-matching areas of parent chromosomes $\mathrm{P}$ and $\mathrm{Q}$, and then exchange the matching area 7-1-3 and 1-7-8 of parent chromosomes $\mathrm{P}$ and $\mathrm{Q}$. So, the progeny chromosomes $\mathrm{p}$ and $\mathrm{q}$ are obtained.

(3) Mutation operation. As all genic values in a chromosome cannot be repeated between each other, it can realize mutation operation by randomly exchanging two genes in the chromosome. This method is called exchange mutation (EM) (Larranaga et al. 1999). The detailed principle of mutation operation is shown in Figure 4. The exchange of gene 8 and 4 in the parent chromosome P' produces the progeny chromosome p.

Step 5. Set termination conditions. The maximum iterative times is used as the termination condition of IGA, and is easily influenced by coding mode, chromosome size, population size, crossover probability, mutation probability, and so on. So, the set value of maximum iterative times requires a comprehensive consideration of various factors. If the set value is too large, it will waste a lot of time; On the contrary, the optimal solution may be missed.

\section{Problem description}

A precast concrete building generally has many floors, and each floor can be divided into some subassembly bodies to simplify its ASPO, such bottom floor slabs, exterior walls, interior walls, etc. By consulting some experts and norms related to prefabricated buildings in China, a precast shear wall structure is usually assembled in the following order: bottom floor slabs, exterior walls, interior walls, beams, top floor slabs and stairs. This is a general principle, and slight adjustments are required for actual projects. However, a subassembly body may still have a large number of constituent components, and it is very difficult to determine the assembly precedence relationship between them.

With reference to the hypothetical panelised structure studied by Shewchuk and Guo (2012), a new precast concrete building model with shear wall structure is created by BIM technology and its plan views are shown in Figure 5. The parametric modelling of BIM is further embodied in this process, which makes BIM very suitable for prefabricated building design. Figures 5(a) and 5(b) show the plan 


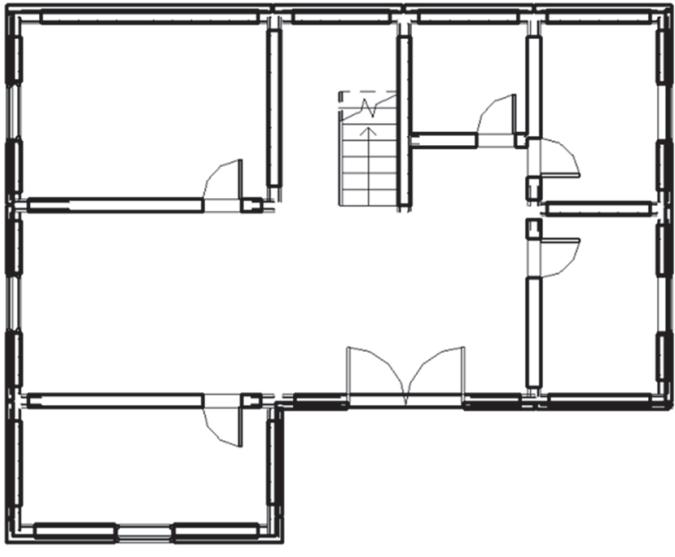

(a) Lower plan view

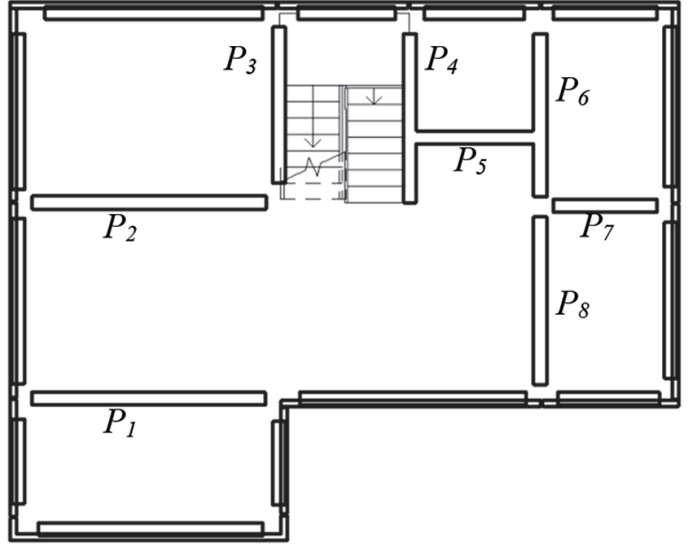

(b) Higher plan view

Figure 5. Plan views of a precast concrete building model

views of a floor from the perspective of different levels, but the bottom floor slabs, beams and top floor slabs are not shown in these plan views. The general assembly process of the building model is bottom floor slabs, exterior walls, interior walls, beams, top floor slabs and stairs. The precast interior walls in Figure 5(b) are marked in turn, but their more detailed assembly sequence cannot be easily determined in comparison with those of other subassembly bodies which can be determined solely by the individual's experience.

Table 1 shows relevant information about these interior walls, including weight relationship, occupied space relationship and interference relationship between precast interior walls. BIM can maintain object information related to precast components. Therefore, some basic data required for Table 1 can be obtained from the BIM model in Figure 5, but some specific calculations still need to be undertaken by relevant personnel. The weight of a precast component itself can also be replaced by its quality. Occupied space is the space occupied by a precast component in its corresponding assembly body. Interference means that a component's installation is hampered by other installed components. All precast components of the building model take the way of vertical lifting and are stacked in the same area, and the sight of a lifting operator is not blocked by installed components. These assumptions reflect current construction practice about precast concrete buildings in China, and greatly draw on some information provided by a prefabricated enterprise in Heilongjiang which is visited by the authors.

\section{Problem solving}

\subsection{Mathematical modelling}

The precast concrete building model in Figure 5 should be assembled in the following general order: bottom floor slabs, exterior walls, interior walls, beams, top floor slabs and stairs. However, a more detailed assembly sequence of precast interior walls in Figure 5 cannot be easily determined. In order to solve this problem, an assembly sequence of precast interior walls in Figure 5 can be expressed as a continuous and non-repeated positive integer string between 1 and 8 . The indices in Table 1 can be used as an evaluation index system of assembly sequence for precast interior walls by reviewing literature and consulting experts. In order to evaluate an assembly sequence quantitatively, the three evaluation indexes in Table 1 are defined as follows.

(1) Weight of components. The greater the weight of a component, the earlier it should be installed. If the assembly of a component violates this principle, a penalty value will be generated. The penalty value can take the weight ratio of this component to the previous installed component. Assume that an assembly body consists of the components $P_{1}, P_{2}, \ldots, P_{n}$. The two-dimensional comparison matrix $A$ which can represent all possible penalty values is shown in Eqn (1). The $a_{i j}$ is any penalty value of the matrix $A$. The $x_{i}$ represents the weight of component $P_{i}$. The $x_{j}$ represents the weight of component $P_{j}$.

Table 1. Relevant information about precast interior walls

\begin{tabular}{|ll|}
\hline \multicolumn{1}{|c|}{ Indices } & \multicolumn{1}{c|}{ Detailed description } \\
\hline Weight & $P_{1}=P_{2}>P_{4}>P_{3}>P_{6}=P_{8}>P_{7}>P_{5}$ \\
& $\left(P_{1}=P_{2}=1.2 P_{4}=1.3 P_{3}=1.5 P_{6}=1.5 P_{8}=2 P_{7}=2.3 P_{5}\right)$ \\
Occupied space & $P_{1}=P_{2}>P_{4}>P_{3}=P_{6}=P_{8}>P_{5}>P_{7}$ \\
& $\left(P_{1}=P_{2}=1.35 P_{4}=1.46 P_{3}=1.46 P_{6}=1.46 P_{8}=2.11 P_{5}=2.25 P_{7}\right)$ \\
Interference & The following orders should not appear as much as possible: \\
& $\left(P_{4}, P_{6}\right)>P_{5}\left(P_{5}, P_{7}\right)>P_{6}$ or $\left(P_{6}, P_{8}\right)>P_{7}$. The ">" here means "precede over" \\
\hline
\end{tabular}




$$
\begin{aligned}
& A=\left(a_{i j}\right)_{n \times n}=\left(\left\{\begin{array}{l}
\frac{x_{j}}{x_{i}}, x_{j}>x_{i} \\
0, x_{j} \leq x_{i}
\end{array}\right)_{n \times n} .\right. \\
& i=1,2, \ldots, n ; j=1,2, \ldots, n .
\end{aligned}
$$

(2) Occupied space of components. The greater the occupied space of a component, the earlier it should be installed. If the assembly of a component violates this principle, a penalty value will be generated. The penalty value can take the occupied space ratio of this component to the previous installed component. Assume that an assembly body consists of the components $P_{1}, P_{2}, \ldots, P_{n}$. The two-dimensional comparison matrix $B$ can represent all possible penalty values, as shown in Eqn (2). The $b_{i j}$ is any penalty value of the matrix $B$. The $y_{i}$ represents the occupied space of component $P_{i}$. The $y_{j}$ represents the occupied space of component $P_{j}$.

$$
\begin{aligned}
& B=\left(b_{i j}\right)_{n \times n}=\left(\left\{\begin{array}{l}
\frac{y_{j}}{y_{i}}, y_{j}>y_{i} \\
0, y_{j} \leq y_{i}
\end{array}\right)_{n \times n} .\right. \\
& i=1,2, \ldots, n ; j=1,2, \ldots, n .
\end{aligned}
$$

(3) Interference between components. The interference should be avoided as much as possible in the assembly process of components. If the installation of a component is hampered by other installed components, a penalty value will be generated. This penalty value can take the installation difficulty ratio of the component under interference and normal conditions, as shown in formula (3). An assembly body consists of the components $P_{1}, P_{2}, \ldots, P_{n}$. The interfered ones of these components are stored in a one-dimensional array $C$. The $c_{k}$ is any penalty value of the array $C$. The $z_{k}$ represents the installation difficulty of the $k^{\text {th }}$ component in the $C$ under interference condition. The $z_{k 0}$ represents the installation difficulty of the $k^{\text {th }}$ component in the $C$ under normal condition. The $m$ represents the number of interfered components.

$$
C=\left(c_{k}\right)_{1 \times m}=\left(\frac{z_{k}}{z_{k 0}}\right)_{1 \times m}, k=1,2, \ldots, m .
$$

There is a certain contradiction between the above three evaluation indexes in the process of assembly sequence planning and optimization process. The weighting method can solve the contradiction. Assume that a onedimensional array $d$ represents an assembly sequence. The $d(i)$, an element of $d$, represent any one component in an assembly sequence. The weights of the three indexes are $u$, $v$ and $w$ respectively. Therefore, the objective function of assembly sequences is shown in Eqn (4). The $g$ means the total difficulty level of assembly. The difficulty level of assembly has a positive correlation with the time and costs of assembly, and has a negative correlation with assembly quality. In order to reduce assembly time and costs and improve assembly quality, the total difficulty level of assem- bly should be as small as possible. Therefore, the assembly sequence which makes the $g$ minimal is the optimal one:

$$
\min g=u \sum_{i=1}^{n-1} a_{d(i) d(i+1)}+v \sum_{i=1}^{n-1} b_{d(i) d(i+1)}+w \sum_{k=1}^{m} c_{k} .
$$

\subsection{IGA-based optimization}

The fitness function generally takes the maximum value in the IGA. The greater the fitness value of an individual, the stronger the individual ability to survive. So, the Eqn (4) can be transformed into a fitness function $f$ by a reciprocal method, as shown in Eqn (5). As the denominator in the Eqn (5) must be not equal to zero, it is necessary to add a nonzero constant $t_{0}$. If population average fitness gradually converges and no longer changes with the increase of iteration times in IGA, then the calculated $f$ is the maximum fitness and its corresponding chromosome is the optimal assembly sequence:

$$
\max f=\frac{1}{u \sum_{i=1}^{n-1} a_{d(i) d(i+1)}+v \sum_{i=1}^{n-1} b_{d(i) d(i+1)}+w \sum_{k=1}^{m} c_{k}+t_{0}}
$$

In order to simplify the calculation of assembly sequence of precast interior walls in Figure 5, all penalty values of interference are set to 2 , and the constant $t_{0}$ is set to 1. The $u, v$ and $w$ are set to $0.25,0.25$ and 0.50 respectively by a quantitative-and-qualitative method. The principle of this method is as follows: First, make the values of the three indicators equal to determine their weights preliminarily; second, make pairwise comparison on these indicators to further adjust their weights; third, take multiple trials with IGA and BIM to further adjust their weights again. The interference between precast components is the least desirable during assembly construction. The weight of interference between components, 0.50 , is the largest between the three indexes. It means that an assembly sequence calculated by IGA gives priority to the interference constraint and then considers the other two constraints. Matlab programming technology is used to realize the IGA. IGA parameters affect each other, and their settings needs to take into account the accuracy and efficiency of calculation. Population size and iteration times are generally positively correlated with chromosome length. After many trials, IGA parameters are set as follows: select elite operation, the length of chromosome is 8 , population size is 200 , iteration times are 1000 , crossover probability is 0.95 , and mutation probability is 0.05 . IGA calculation results are as follows: an optimal assembly sequence of precast interior walls in Figure 5 is 1, 2, 3, 6, 5, 7, 4 and 8, and the cumulative penalty values of weight, occupied space, and interference are separately $2.8167,1.6667$, and 0 . The optimal fitness value is 0.4715 . So, the objective function value is 1.1209. Figure 6 shows the relationships between population average fitness and iteration times, and the calculation results have good convergence. This means that the IGA experiment arrives at maturity before its termina- 


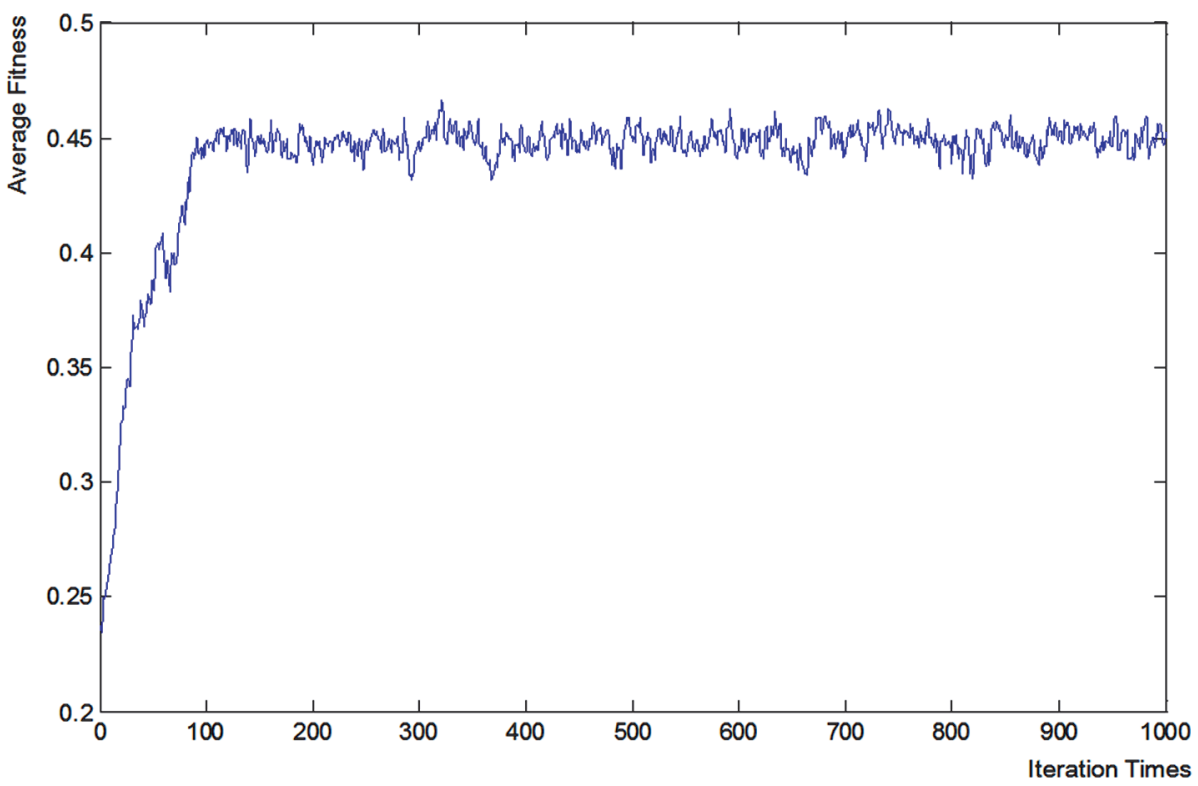

Figure 6. Relationships between population average fitness and iteration times

tion. The best individual first appears in the fifteenth generation. The optimal assembly sequence of precast interior walls in Figure 5 is not unique, and other optimal assembly sequences are no longer listed here one by one.

\subsection{BIM-based visual simulation}

BIM is a popular technology in the construction industry, and its parametric modelling coincides with assembly construction of prefabricated buildings (Moya, Pons 2014; Zhong et al. 2017; Lin et al. 2016). Therefore, BIMbased visual simulation technology is used to simulate the construction process of a precast concrete building. An assembly sequence generated by IGA can be further checked by visual simulation. If there are some unreasonable phenomena, some parameters of the above mathematical models can be adjusted to further optimize the assembly sequence. Take the optimal assembly sequence $1,2,3,6,5,7,4$ and 8 of precast interior walls in Figure 5 as an example, and BIM-based visual simulation is shown in Figure 7. Compared with two-dimensional drawings, BIM model, a three-dimensional model, is more intuitive.
The individual's experience knowledge is combined with the visual simulation in Figure 7, which makes it easier to find out whether the assembly sequence is unreasonable. The simulation process indicates that there is no unreasonable installation of precast interior walls. The precast concrete building model in Figure 5 should be assembled in the following detailed order: bottom floor slabs, exterior walls, interior walls (1, 2, 3, 6, 5, 7, 4 and 8), beams, top floor slabs and stairs.

\subsection{Scheme dynamic adjustment}

The assembly order of one or two precast components may be wrong for some reason in actual construction process. Once this phenomenon occurs, it is necessary to adjust the subsequent assembly sequence in a timely manner. The optimal assembly sequence $1,2,3,6,5,7$, 4 and 8 of precast interior walls in Figure 5 is till taken as an example, and the component 3 is assembled before the component 2, as shown in Figure 8. This disrupts the subsequent assembly sequence.
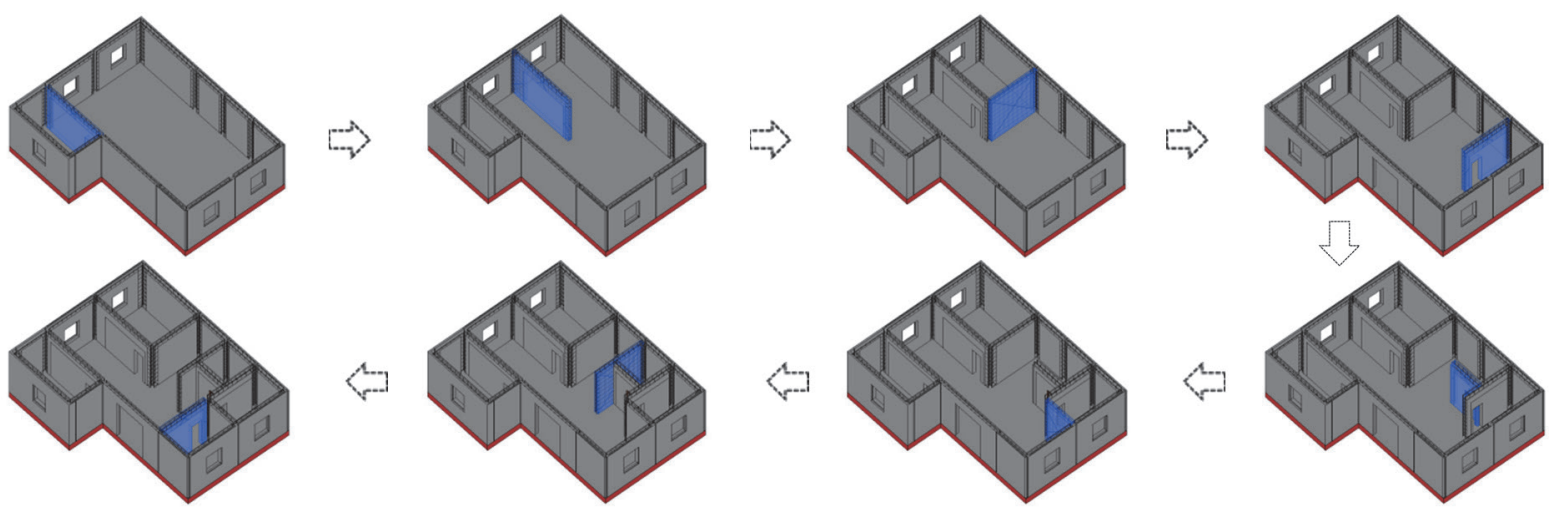

Figure 7. BIM-based visual simulation about testing an assembly sequence 


\begin{tabular}{c|c|c|c|c|c|c|c|c|} 
Original sequence & 1 & 2 & 3 & 6 & 5 & 7 & 4 & 8 \\
\hline
\end{tabular} Possible sequence \begin{tabular}{|l|l|l|l|l|l|l|l|}
\hline 1 & 3 & $?$ & $?$ & $?$ & $?$ & $?$ & $?$ \\
\hline
\end{tabular}

Figure 8. Example of assembly order errors

The positions of components 1 and 3 in any possible sequence have been determined and cannot be changed. But, the locations of other components have not yet been determined and need to be further optimized. The IGA is still used to solve this situation, and the settings of the relevant parameters remain unchanged. The results are as follows: an optimal assembly sequence of precast interior walls in Figure 5 is 1, 3, 6, 5, 7, 2, 4 and 8, and the cumulative penalty values of weight, occupied space, and interference are separately $3.1500,2.2500$, and 0 . The optimal fitness value is 0.4255 . So, the objective function value is 1.3500. Figure 9 shows the relationships between population average fitness and iteration times, and the calculation results have good convergence. This means that the IGA experiment arrives at maturity before its termination. The best individual first appears in the ninth generation.

If time permits in actual construction process, BIMbased visual simulation technology should be used again to further check the rationality of the assembly sequence $1,3,6,5,7,2,4$ and 8 of precast interior walls in Figure 5, as shown in Figure 10. The simulation process reflects the contradictory relationship between weight, occupied space, and interference of an assembly sequence. In order to avoid interference between components as much as possible, the component 2 is not given priority although its weight and occupied space are the largest. However, this sequence is optimal and reasonable from the perspective of comprehensively considering the three indexes. The precast concrete building model in Figure 5 should continue to be assembled in the following new order: bottom floor slabs, exterior walls, interior walls $(1,3,6,5,7,2,4$ and 8), beams, top floor slabs and stairs.

\section{Discussion and conclusions}

The BIM-IGA-based ASPO method is established to better solve the ASPO problem in precast concrete buildings. BIM in the BIM-IGA-based ASPO method is mainly used to draw a prefabricated building model parametrically and simulate its assembly process visually. IGA in the BIM-IGA-based ASPO method is used to calculate some mathematical models of evaluating assembly sequences, and generates an optimal assembly sequence. This optimal assembly sequence also needs to be further tested and optimized by BIM's visual simulation. In addition, BIM's visual simulation can also train construction workers in advance and minimize the assembly sequence errors caused by individual reasons during construction. BIM and IGA work together organically in the BIM-IGAbased ASPO method.

The BIM-IGA-based ASPO method has some premise assumptions which are derived from the construction practice of most precast concrete buildings in reality. If these assumptions are changed, an evaluation index system may contain more indexes than the weight, occupied space and interference. For different precast concrete

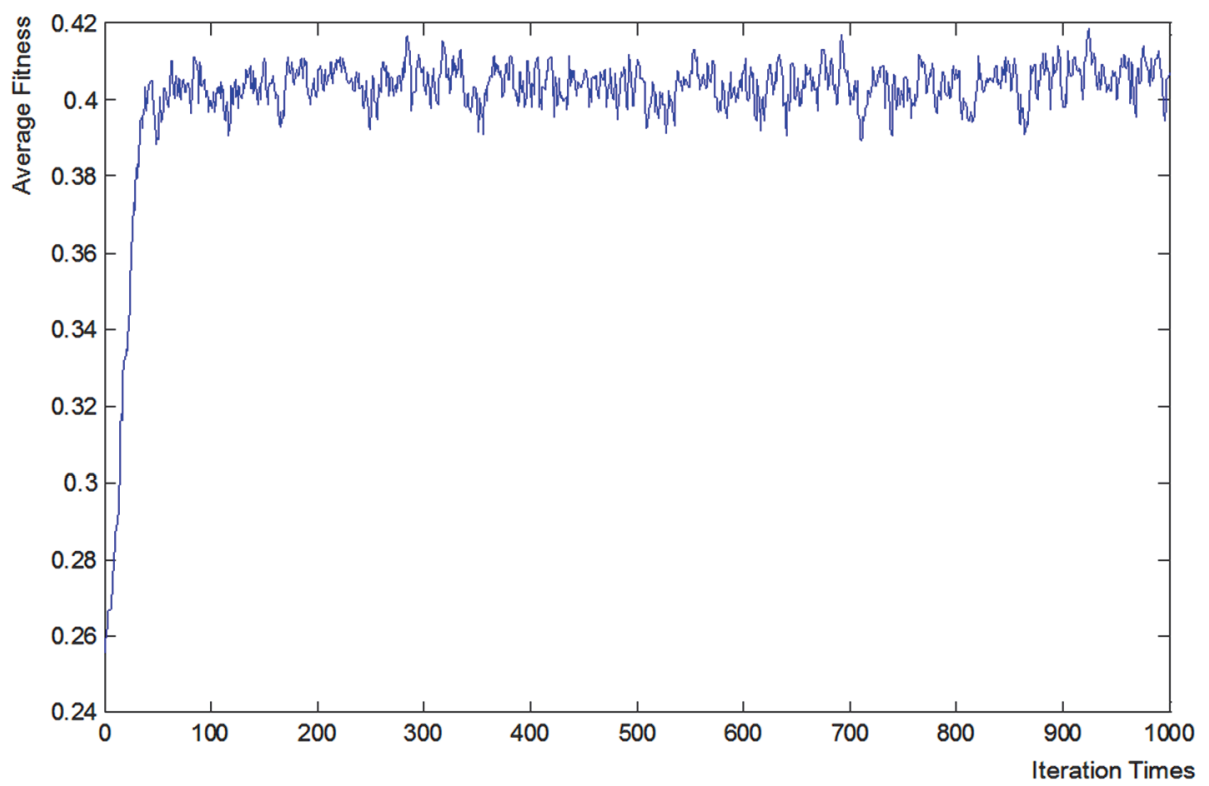

Figure 9. Relationships between population average fitness and iteration times under dynamic adjustment 

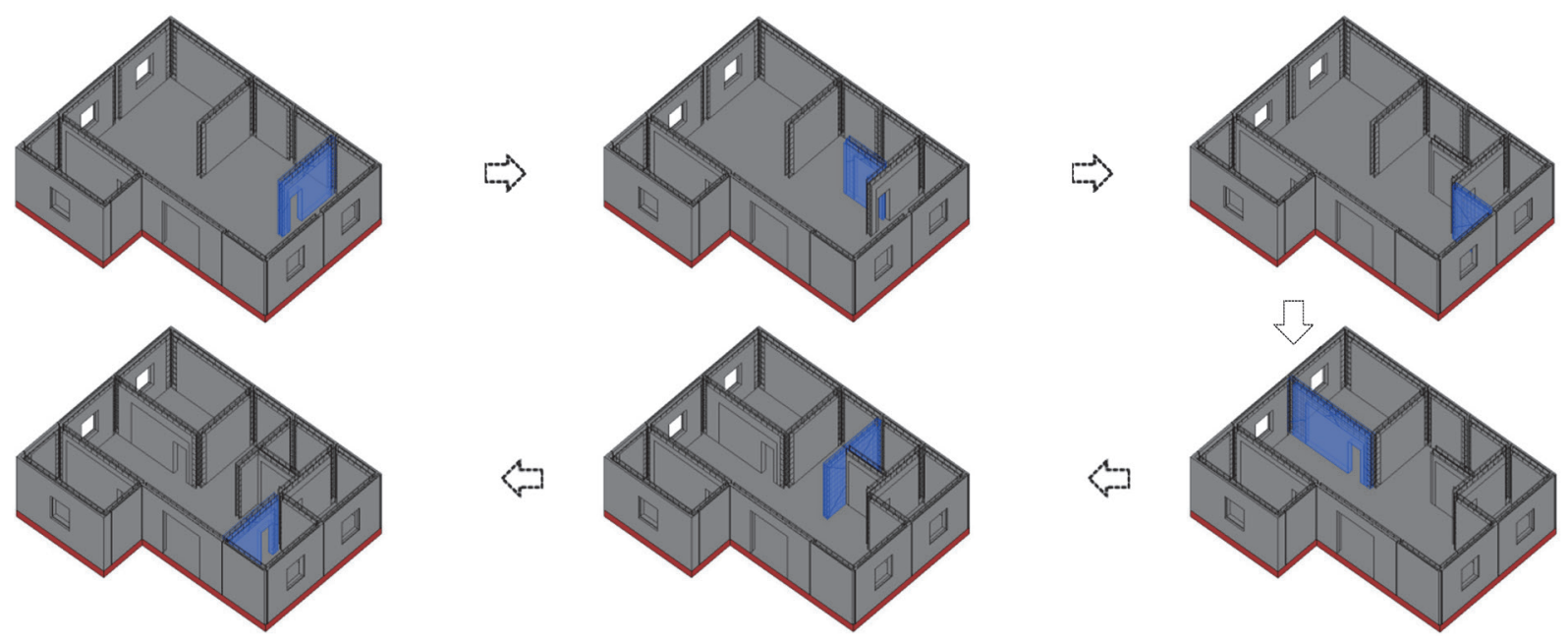

Figure 10. BIM-based visual simulation in construction process

buildings, the weights between weight, occupied space and interference are not constant and need to be fine-tuned. Therefore, the objective function of evaluating assembly sequences also need to be adjusted correspondingly. Realnumber encoding, SPMCM, and exchange mutation (EM) method are introduced or developed in the IGA to evaluate and select an optimal assembly sequence. The example results in the section 3.2 of this paper show that more than one optimal assembly sequence is obtained in the calculation process, and decision makers can select one of them as a planning scheme. Sometimes, the IGA is likely to fall into the local optimal solution. This means that an assembly sequence obtained by IGA is not necessarily the best. However, this phenomenon can be reduced by increasing the population size, iteration times, cross probability and mutation probability of IGA.

This paper also studies how to dynamically adjust an assembly sequence in construction process, and the BIMIGA-based ASPO method is again proven to solve this problem well. However, the example results in this paper show that when the number of precast components is relatively large, the BIM-IGA-based ASPO method is no longer a highly efficient method. Besides, this paper does not consider too much the on-site stacking and identification of precast components. These issues are conducive to perfecting the knowledge system related to the assembly order of precast concrete buildings, and will be further research directions.

\section{Acknowledgements}

Sincerely thank a precast concrete enterprise in Heilongjiang Province for providing relevant information and guidance. The research is supported by the National Key Research and Development Program of China (grant number 2016YFC0701900) and the National Natural Science Foundation of China (grant number 51378160).

\section{Disclosure statement}

The authors in this paper have no competing financial, professional, or personal interests from other parties.

\section{References}

Alcaraz, J.; Maroto, C. 2001. A robust genetic algorithm for resource allocation in project scheduling, Annals of Operations Research 102(1-4): 83-109. https://doi.org/10.1023/A:1010949931021

Aldwaik, M.; Adeli, H. 2014. Advances in optimization of highrise building structures, Structural and Multidisciplinary Optimization 50(6): 899-919. https://doi.org/10.1007/s00158-014-1148-1

Boafo, F. E.; Kim, J.-H.; Kim, J.-T. 2016. Performance of modular prefabricated architecture: case study-based review and future pathways, Sustainability 8: 558.

https://doi.org/10.3390/su8060558

de Oliveira, R. A.; de Medeiros, Jr., M. F.; Menezes, R. F. A. 2014. Application of genetic algorithm for optimization on projects of public illumination, Electric Power Systems Research 117: 84-93. https://doi.org/10.1016/j.epsr.2014.08.008

Ding, J.; Hu, K.; Chen, Y.; Qu, G.; Li, Q.; Xiao, B. 2016. Discussion on the precast rate of monolithic precast concrete shear wall structure, Building Structure 46: 628-632 (in Chinese).

Dong, T.; Tong, R.; Zhang, L.; Dong, J. 2007. A knowledge-based approach to assembly sequence planning, International Journal of Advanced Manufacturing Technology 32(11-12): 12321244. https://doi.org/10.1007/s00170-006-0438-1

Fung, I. W. H.; Huang, C.; Tam, C. M. 2013. Application of GA optimization for solving precedent relationship problem in project scheduling, Mathematical and Computer Modelling 57(9-10): 2067-2081. https://doi.org/10.1016/j.mcm.2011.06.022

Gao, L.; Qian, W.; Li, X.; Wang, J. 2010. Application of memetic algorithm in assembly sequence planning, International Journal of Advanced Manufacturing Technology 49(9-12): 11751184. https://doi.org/10.1007/s00170-009-2449-1

Gao, Z.; Xue, S.; He, Y. 2015. Analysis of design and construction integration of rigid bracing dome, Advances in Structural Engineering 18(11): 1947-1958.

https://doi.org/10.1260/1369-4332.18.11.1947 
Groba, C.; Sartal, A.; Vazquez, X. H. 2015. Solving the dynamic traveling salesman problem using a genetic algorithm with trajectory prediction: An application to fish aggregating devices, Computers \& Operations Research 56: 22-32. https://doi.org/10.1016/j.cor.2014.10.012

Han, G.; Srebric, J.; Enache-Pommer, E. 2014. Variability of optimal solutions for building components based on comprehensive life cycle cost analysis, Energy and Buildings 79: 223-231. https://doi.org/10.1016/j.enbuild.2013.10.036

Hu, W. 2007. Analysis of construction process planning based on geometric reasoning, Journal of Tongji University (Natural Science) 35(4): 566-570 (in Chinese).

Iwankowicz, R. R. 2016. An efficient evolutionary method of assembly sequence planning for shipbuilding industry, Assembly Automation 36(1): 60-71. https://doi.org/10.1108/AA-02-2015-013

Jiang, Q. 2010. Summary on development of assembled concrete building both home and abroad, Architecture Technology 41(12): 1074-1077 (in Chinese).

Johnston, B.; Bulbul, T.; Beliveau, Y.; Wakefield, R. 2016. An assessment of pictographic instructions derived from a virtual prototype to support construction assembly procedures, Automation in Construction 64: 36-53. https://doi.org/10.1016/j.autcon.2015.12.019

Larranaga, P.; Kuijpers, C. M. H.; Murga, R. H.; Inza, I.; Dizdarevic, S. 1999. Genetic algorithms for the travelling salesman problem: a review of representations and operators, Artificial Intelligence Review 13(2): 129-170.

https://doi.org/10.1023/A:1006529012972

Li, P.; Cui, J.; Gao, F.; Wang, C.; Mao, Y.; Liao, G. 2015. Research on the assembly sequence of a ship block based on the disassembly interference matrix, Journal of Ship Production and Design 31(4): 230-240.

https://doi.org/10.5957/JSPD.31.4.140009

Li, S.-H. 2012. Embodied environmental burdens of wood structure in Taiwan compared with reinforced concrete and steel structures with various recovery rates, Applied Mechanics and Materials 174-177: 202-210. https://doi.org/10.4028/www. scientific.net/AMM.174-177.202

Lin, Y.-C.; Lee, H.-Y; Yang, I.-T. 2016. Developing as-built BIM model process management system for general contractors: A case study, Journal of Civil Engineering and Management 22(5): 608-621. https://doi.org/10.3846/13923730.2014.914081

Liu, P.; Li, Q.; Song, L.; Jia, R. 2017. The index system for the development level evaluation of regional construction industrialization: a case study in Jiangsu, China, Applied Sciences 7(5). https://doi.org/10.3390/app7050492

Manrique, J. D.; Al-Hussein, M.; Telyas, A.; Funston, G. 2007. Constructing a complex precast tilt-up-panel structure utilizing an optimization model, 3D CAD, and animation, Journal of Construction Engineering and Management 133(3): 199-207. https://doi.org/10.1061/(ASCE)0733-9364(2007)133:3(199)

Moya, Q.; Pons, O. 2014. Improving the design and production data flow of a complex curvilinear geometric glass reinforced concrete façade, Automation in Construction 38: 46-58. https://doi.org/10.1016/j.autcon.2013.10.025

Qu, S.; Jiang, Z.; Tao, N. 2013. An integrated method for block assembly sequence planning in shipbuilding, International Journal of Advanced Manufacturing Technology 69(5-8): 1123-1135. https://doi.org/10.1007/s00170-013-5087-6
Senouci, A.; Al-Derham, H. R. 2008. Genetic algorithm-based multi-objective model for scheduling of linear construction projects, Advances in Engineering Software 39(12): 1023-1028. https://doi.org/10.1016/j.advengsoft.2007.08.002

Shan, H.; Zhou, S.; Sun, Z. 2009. Research on assembly sequence planning based on genetic simulated annealing algorithm and ant colony optimization algorithm, Assembly Automation 29(3): 249-256. https://doi.org/10.1108/01445150910972921

Shewchuk, J. P.; Guo, C. 2012. Panel stacking, panel sequencing, and stack locating in residential construction: lean approach, Journal of Construction Engineering and Management 138(9): 1006-1016. https://doi.org/10.1061/(ASCE)CO.1943-7862.0000520

Tezel, A.; Nielsen, Y. 2013. Lean construction conformance among construction contractors in Turkey, Journal of Management in Engineering 29(3): 236-250.

https://doi.org/10.1061/(ASCE)ME.1943-5479.0000145

Tseng, Y.-J.; Kao, H.-T.; Huang, F.-Y. 2010. Integrated assembly and disassembly sequence planning using a GA approach, International Journal of Production Research 48(20): 59916013. https://doi.org/10.1080/00207540903229173

Wang, N.; Chang, Y.-Z. 2004. Application of the genetic algorithm to the multi-objective optimization of air bearings, Tribology Letters 17(2): 119-128. https://doi.org/10.1023/B:TRIL.0000032436.09396.d4

Wang, Y.; Liu, J. H. 2010. Chaotic particle swarm optimization for assembly sequence planning, Robotics and ComputerIntegrated Manufacturing 26(2): 212-222. https://doi.org/10.1016/j.rcim.2009.05.003

Zabihi, H.; Habib, F.; Mirsaeedie, L. 2013. Definitions, concepts and new directions in Industrialized Building Systems (IBS), KSCE Journal of Civil Engineering 17(6): 1199-1205. https://doi.org/10.1007/s12205-013-0020-y

Zhao, Z.; Zhu, H.; Chen, Z.; Du, Y. 2015. Optimizing the construction procedures of large-span structures based on a real-coded genetic algorithm, International Journal of Steel Structures 15(3): 761-776. https://doi.org/10.1007/s13296-015-9020-8

Zhong, R. Y.; Peng, Y.; Xue, F.; Fang, J.; Zou, W.; Luo, H.; Ng, S. T.; Lu, W.; Shen, G. Q. P.; Huang, G. Q. 2017. Prefabricated construction enabled by the Internet-of-Things, Automation in Construction 76: 59-70.

https://doi.org/10.1016/j.autcon.2017.01.006 